\title{
New inequalities for star bodies
}

\author{
Yanxiong Yan ${ }^{1}$, Liangcai Zhang ${ }^{2}$ and Min Zhou ${ }^{3 *}$
}

"Correspondence:
zm723@swu.edu.cn
${ }^{3}$ Department of Financial,
Southwest University, Beibei,
Chongqing, 400715, P.R. China
Full list of author information is
available at the end of the article

${ }^{\text {*Correspondence: }}$ zm723@swu.edu.cn Southwest University, Beibei, Chongqing, 400715, P.R. China available at the end of the article

\begin{abstract}
In this paper, we investigate the radial addition and Blaschke addition and get some new Brunn-Minkowski inequalities associated with dual quermassintegrals and chord integral for star bodies.

MSC: 52A20; 52A40

Keywords: Brunn-Minkowski theory; radial addition; dual Brunn-Minkowski theory; mixed volumes; Blaschke addition
\end{abstract}

\section{Introduction}

The Brunn-Minkowski theory, which is the so-called mixed-volume theory, is the classical core of the geometry of convex bodies. This theory originated from the thesis of Hermann Brunn in 1887, and in its essential part is the creation of Hermann Minkowski around the turn of the century. The well-known survey of Bonnesen and Fenchel collected an impressive body of results in 1934, though important developments, by the work of Aleksandrov and others in the $1930 \mathrm{~s}$, were still to come. In recent years, the theory of convex bodies was expanded considerably, new topics have been developed rapidly, and originally neglected branches of the subject have gained in interest. For example, the Brunn-Minkowski theory has remained of constant interest owing to its various new applications and connections with other fields.

The classical Brunn-Minkowski theory forms a central part of Brunn-Minkowski theory of convex bodies and arises naturally if one combines the two fundamental concepts of Minkowski addition and volume (see [1, 2]). The famous Brunn-Minkowski inequality implies that, for two convex bodiesin the Euclidean space $\mathbb{R}^{n}$ (see [3]),

$$
V(K+L)^{\frac{1}{n}} \geq V(K)^{\frac{1}{n}}+V(L)^{\frac{1}{n}}
$$

where $V$ denotes the volume, with inequality if and only if $K$ and $L$ are homothetic. This geometric inequality means that if each of $K, L$ has volume 1 , then the Minkowski sum $\frac{1}{2}(K+L)$ has volume at least 1 , and its volume is equal to 1 only if $K$ and $L$ are translates.

During the last three decades, the Brunn-Minkowski theory has achieved important developments. In the 1970s, Lutwak's dual Brunn-Minkowski theory had come out, which helped to achieve major breakthrough of solving the Busemann-Petty problem in the 1990s. In the dual theory, compared with the Brunn-Minkowski theory, convex bodies are replaced by star-shaped bodies, and projections onto subspaces are replaced by inter-

(c) 2016 Yan et al. This article is distributed under the terms of the Creative Commons Attribution 4.0 International License (http://creativecommons.org/licenses/by/4.0/), which permits unrestricted use, distribution, and reproduction in any medium, provided you give appropriate credit to the original author(s) and the source, provide a link to the Creative Commons license, and indicate if changes were made. 
sections with subspaces. The machinery of the dual theory includes dual mixed volumes and intersection bodies (see [1, 2, 4-12]).

The radial addition and Blaschke addition are still playing a crucial role in the BrunnMinkowski theory. In this article, we continue to investigate the radial addition and Blaschke addition and get some new Brunn-Minkowski inequalities associated with dual quermassintegrals and chord integral for star bodies.

\section{Preliminaries}

A set $K$ of points in the Euclidean space $\mathbb{R}^{n}$ is convex if for any $x, y \in K$, we have $0 \leq \lambda \leq 1$ and $\lambda x+(1-\lambda) y \in K$. A domain is a set with nonempty interiors. A convex body is a compact convex domain. The set of convex bodies in $\mathbb{R}^{n}$ is denoted by $\mathcal{K}^{n}$. Let $\mathcal{K}_{o}^{n}$ be the class of members of $\mathcal{K}^{n}$ containing the origin in their interiors. We write $V$ for the $n$ dimensional Lebesgue measure and $\mathcal{H}^{n-1}$ for the $(n-1)$-dimensional Hausdorff measure. We denote by $S^{n-1}$ the surface of the unit ball in $\mathbb{R}^{n}$.

A convex body $K \subset \mathbb{R}^{n}$ is uniquely determined by its support function $h_{K}: \mathbb{R}^{n} \rightarrow \mathbb{R}$, where $h_{K}(x)=\max \{x \cdot y: y \in K\}$ for $x \in \mathbb{R}^{n}$. The support function of the dilate $c K=\{c x$ : $x \in K\}$ of a convex body $K$ satisfies the equality

$$
h_{c K}=c h_{K},
$$

where $c>0$. Note that support functions are positively homogeneous of degree one and subadditive. It follows immediately from the definition of support functions that, for convex bodies $K$ and $L$, we have

$$
K \subseteq L \quad \Longleftrightarrow h_{K} \leq h_{L}
$$

For a convex body $K$ and each Borel set $\omega \subset S^{n-1}$, the reverse spherical image $\tau(K, \omega)$ of $K$ at $\omega$ is the set of all boundary points of $K$ that have their outer unit normal belonging to the set $\omega$. Associated with each convex body $K \in \mathcal{K}_{o}^{n}$, there is a Borel measure $S_{K}$ on $S^{n-1}$, called the Aleksandrov-Fenchel surface area measure of $K$, which is defined as

$$
S_{K}(\omega)=\mathcal{H}^{n-1}(\tau(K, \omega))
$$

for each Borel set $\omega \subseteq \mathbb{S}^{n-1}$. For the surface area measure of the dilate $c K$ of $K$, we have

$$
S_{c K}=c^{n-1} S_{K}
$$

where $c>0$. The Minkowski sum of convex sets $K_{1}, \ldots, K_{m}$ can be defined as

$$
K_{1}+\cdots+K_{m}=\left\{y_{1}+\cdots+y_{m}: y_{1} \in K_{1}, \ldots, y_{m} \in K_{m}\right\} .
$$

By the definition of support function we have

$$
h\left(\lambda_{1} K_{1}+\cdots+\lambda_{r} K_{r}, \cdot\right)=\lambda_{1} h\left(K_{1}, \cdot\right)+\cdots+\lambda_{r} h\left(K_{r}, \cdot\right) .
$$


The mixed volume $V\left(K_{1}, \ldots, K_{n}\right)$ of compact convex sets $K_{1}, \ldots, K_{n}$ is defined by

$$
V\left(K_{1}, \ldots, K_{n}\right)=\frac{1}{n !} \sum_{j=1}^{n}(-1)^{n+j} \sum_{i_{1}<\cdots<i_{k}} V\left(K_{i_{1}}+\cdots+K_{i_{k}}\right) .
$$

The radial function $\rho_{M}=\rho(M, \cdot): \mathbb{R}^{n} \backslash\{0\} \rightarrow[0, \infty)$ of a compact star-shaped (about the origin) $M \subset \mathbb{R}^{n}$ can be defined as (see $[3,4]$ )

$$
\rho(M, y)=\max \{\lambda \geq 0: \lambda y \in M\}
$$

We call $M$ a star body (about the origin) if $\rho_{M}$ is positive and continuous. We write $\mathcal{S}_{o}^{n}$ for the set of star bodies about the origin in $\mathbb{R}^{n}$. Two star bodies $M$ and $N$ are dilates (of one another) if $\rho_{M}(u) / \rho_{N}(u)$ is independent of $u \in S^{n-1}$. For $s>0$, we have

$$
\rho(s M, y)=s \rho(M, y) \quad \text { for all } x \in \mathbb{R}^{n} \backslash\{0\} .
$$

The radial Minkowski addition and scalar product of the sets $M_{1}, \ldots, M_{r} \in \mathcal{S}_{o}^{n}$ and $\lambda_{1}, \ldots, \lambda_{r} \in \mathbb{R}$ is defined by (see $\left.[4,10]\right)$

$$
\lambda_{1} M_{1} \tilde{+} \cdots \tilde{+} \lambda_{r} M_{r}=\left\{\lambda_{1} y_{1} \tilde{+} \cdots \tilde{+} \lambda_{r} y_{r}: y_{i} \in K_{i}, i=1,2, \ldots, r\right\} .
$$

For $M, N \in \mathcal{S}_{o}^{n}$ and $\lambda, \mu \geq 0, a M \tilde{+} b N$ can be defined as the star body such that

$$
\rho_{\lambda M \tilde{+} \mu N}(u)=\lambda \rho_{M}(u)+\mu \rho_{N}(u) \quad \text { for all } u \in S^{n-1} .
$$

The volume formula of a compact set $M$ can be represented by the polar coordinate as follows:

$$
V(M)=\frac{1}{n} \int_{S^{n-1}} \rho_{M}^{n}(u) d S(u),
$$

where $S$ is the Lebesgue measure on $S^{n-1}$ (i.e., the $(n-1)$-dimensional Hausdorff measure).

For $M_{1}, \ldots, M_{r} \in \mathcal{S}_{o}^{n}$ and $\lambda_{1}, \ldots, \lambda_{r} \geq 0$, the volume of $\lambda_{1} M_{1} \tilde{+} \cdots \tilde{+} \lambda_{r} M_{r}$ is defined by

$$
V\left(\lambda_{1} M_{1} \tilde{+} \cdots \tilde{+} \lambda_{r} M_{r}\right)=\sum \tilde{V}_{i_{1}, \ldots, i_{n}}\left(M_{1}, \ldots, M_{r}\right) \lambda_{i_{1}} \cdots \lambda_{i_{n}}
$$

where $\tilde{V}_{i_{1}, \ldots, i_{n}}\left(M_{1}, \ldots, M_{r}\right)$ is the dual mixed volume of $M_{i_{1}}, \ldots, M_{i_{n}}$; we also denote

$$
\tilde{V}\left(M_{i_{1}}, \ldots, M_{i_{n}}\right)=\int_{S^{n-1}} \rho\left(M_{1}, u\right) \cdots \rho\left(M_{n}, u\right) d S(u) .
$$

Let $M_{1}=\cdots=M_{n-1}=M$ and $M_{n-i+1}=\cdots=M_{n}=N$. Then

$$
\tilde{V}(\underbrace{M, \ldots, M}_{n-i}, \underbrace{N, \ldots, N}_{i})
$$

is written as $\tilde{V}_{i}(M, N)$, and so

$$
\tilde{V}_{i}(M, N)=\int_{S^{n-1}} \rho(M, u)^{n-i} \rho(N, u)^{i} d S(u) .
$$


Let $N$ be the unit ball, then $\tilde{V}_{i}(M, B)$ becomes to the dual quermassintegral $\tilde{W}_{i}(M)$, and the last formula implies that

$$
\tilde{W}_{i}(M)=\int_{S^{n-1}} \rho(M, u)^{n-i} d S(u) .
$$

By the dual Minkowski inequality we can obtain the dual Brunn-Minkowski inequality (see [10]):

For $M, N \in \mathcal{S}_{o}^{n}$ and $\lambda, \mu \geq 0$, we have

$$
V(\lambda M \tilde{+} \mu N)^{\frac{1}{n}} \leq \lambda V(M)^{\frac{1}{n}}+\mu V(N)^{\frac{1}{n}}
$$

with equality if and only if $M$ and $N$ are dilates.

For $K, L \in \mathcal{K}^{n}$, by the solution of the Minkowski problem, there exists a convex body $C$ such that

$$
S(C, \cdot)=S(K, \cdot)+S(L, \cdot) .
$$

The body $C$, denoted by $K \sharp L$, is called the Blaschke sum of $K$ and $L$.

For Blaschke addition, a counterpart to the Brunn-Minkowski inequality, is the following:

$$
V(K \sharp L)^{\frac{n-1}{n}} \geq V(K)^{\frac{n-1}{n}}+V(L)^{\frac{n-1}{n}}
$$

with equality if and only if $K$ and $L$ are homothetic.

For $M \in \mathcal{S}_{o}^{n}$, the half-chord along the direction $u \in \mathbb{S}^{n}-1$ passing through $y \in M$, $p_{M}(x, u)$, is defined by

$$
p_{M}(y, u)=\frac{1}{2}\left(\rho_{M}(y, u)+\rho_{M}(y,-u)\right) .
$$

Then the chord integral of $M$ can be defined as

$$
P_{i}(M ; y)=\frac{1}{n} \int_{S^{n-1}} p_{M}(y, u)^{n-i} d S(u) .
$$

For $M, N \in \mathcal{S}_{o}^{n}$ and $y \in M \cap N$, it is easy to see that

$$
p_{M \tilde{+} N}(y, u)=p_{M}(y, u)+p_{N}(y, u) .
$$

We also need the following Minkowski inequality for integrals is needed (see [13]):

Let $f, g: \mathbb{S}^{n-1} \longrightarrow \mathbb{R}$ be positive continuous functions, and let $1<p<\infty$. Then we have

$$
\left(\int_{\mathbb{S}^{n-1}}(f+g)^{p} d \sigma\right)^{\frac{1}{p}} \leq\left(\int_{\mathbb{S}^{n-1}} f^{p} d \sigma\right)^{\frac{1}{p}}+\left(\int_{\mathbb{S}^{n-1}} g^{p} d \sigma\right)^{\frac{1}{p}},
$$

where equality holds if and only if $f$ and $g$ are proportional.

If $p<0$ or $0<p<1$, the inequality is reverse. 


\section{Inequalities of dual quermassintegrals}

Theorem 1 For $M, N \in \mathcal{S}_{o}^{n}$, we have $\tilde{W}_{2 n}(M)^{\frac{1}{n}}+\tilde{W}_{2 n}(N)^{\frac{1}{n}} \geq 4 \tilde{W}_{2 n}(M \tilde{+} N)^{\frac{1}{n}}$ with equality if and only if $M$ and $N$ are the same.

Proof By the Minkowski inequality for integrals we have that

$$
\begin{aligned}
\tilde{W}_{2 n}(M)^{\frac{1}{n}}+\tilde{W}_{2 n}(N)^{\frac{1}{n}} & =\left(\frac{1}{n} \int_{S^{n}}\left(\frac{1}{\rho(M, u)}\right)^{n} d S(u)\right)^{\frac{1}{n}}+\left(\frac{1}{n} \int_{S^{n}}\left(\frac{1}{\rho(N, u)}\right) d S(u)\right)^{\frac{1}{n}} \\
& \geq\left(\frac{1}{n} \int_{S^{n}}\left(\frac{1}{\rho(M, u)}+\frac{1}{\rho(N, u)}\right)^{n} d S(u)\right)^{\frac{1}{n}} \\
& \geq 4\left(\frac{1}{n} \int_{S^{n}}\left(\frac{1}{\rho(M, u)+\rho(N, u)}\right)^{n} d S(u)\right)^{\frac{1}{n}} \\
& =4\left(\frac{1}{n} \int_{S^{n}}\left(\frac{1}{\rho(M \tilde{+} N, u)}\right)^{n} d S(u)\right)^{\frac{1}{n}} \\
& =4 \tilde{W}_{2 n}(M \tilde{+} N)^{\frac{1}{n}}
\end{aligned}
$$

with equality if and only if $\rho(M, u)=\rho(N, u)$, that is, $M$ and $N$ are the same.

For Blaschke addition, we have the following theorem.

Theorem 2 Let $K_{j}(j=1, \ldots, m) \in \mathcal{K}_{o}^{n}$. Then, for $i>n$ or $1<i<n$, we have

$$
W_{i}\left(K_{1} \sharp \cdots \sharp K_{n}\right)^{\frac{n-1}{n-i}} \geq \sum_{j=1}^{m} W_{i}\left(K_{j}\right)^{\frac{n-1}{n-i}}
$$

with equality if and only if $K_{1}, \ldots, K_{m}$ are homothetic.

For $i<1$, we have

$$
\left(\sum_{j=1}^{m} W_{i}\left(K_{j}\right)\right)^{\frac{n-1}{n-i}} W_{i}\left(K_{1} \sharp \cdots \sharp K_{n}\right)^{\frac{n-1}{n-i}} \leq \sum_{j=1}^{m} W_{i}\left(K_{j}\right)^{\frac{n-1}{n-i}}
$$

with equality if and only if $K_{1}, \ldots, K_{m}$ are homothetic.

Proof If $i>n$ or $1<i<n$, then by the definition of Blaschke linear combination and the Minkowski inequality for integrals we can get

$$
\begin{aligned}
W_{i}\left(K_{1} \sharp \cdots \sharp K_{n}\right)^{\frac{n-1}{n-i}} & =\left(\frac{1}{n} \int_{S^{n-1}} \rho\left(K_{1} \sharp \cdots \sharp K_{n}, u\right)^{n-i} d S(u)\right)^{\frac{n-1}{n-i}} \\
& =\left(\frac{1}{n} \int_{S^{n-1}}\left(\left(\rho\left(K_{1}, u\right)^{n-1}+\cdots+\rho\left(K_{n}, u\right)^{n-1}\right)^{n-i}\right)^{\frac{n-i}{n+1}} d S(u)\right)^{\frac{n+1}{n-i}} \\
& \geq \sum_{j=1}^{m}\left(\frac{1}{n} \int_{S^{n-1}}\left(\rho\left(K_{j}, u\right)^{n-i} d S(u)\right)\right)^{\frac{n-1}{n-i}} \\
& =\sum_{j=1}^{m} W_{i}\left(K_{j}\right)^{\frac{n-1}{n-i}},
\end{aligned}
$$


where equality holds (by Minkowski's inequality for integrals) if $K_{1}, \ldots, K_{m}$ are homothetic.

Similarly, for $i<1$, we can get that the reverse inequality of Minkowski's inequality for integrals.

Particularly, if $i=0$, then Theorem 2 implies the following:

Corollary 3.1 Let $K_{j}(j=1, \ldots, m) \in \mathcal{S}_{o}^{n}$. Then

$$
V\left(K_{1} \sharp \cdots \sharp K_{n}\right)^{\frac{n-1}{n}} \leq \sum_{j=1}^{m} V\left(K_{j}\right)^{\frac{n-1}{n}}
$$

with equality if and only if $K_{1}, \ldots, K_{m}$ are homothetic.

\section{Inequalities of chord integral of the star body}

Theorem 3 Let $M, N \in \mathcal{S}_{o}^{n}, y \in K \cap L$. Then

$$
P_{2 n}(M ; y)^{\frac{n-1}{n}}+P_{2 n}(N ; y)^{\frac{n-1}{n}} \geq 4 P_{2 n}(M \tilde{+} N ; y)^{\frac{n-1}{n}}
$$

with equality if and only if $M$ and $N$ are the same.

Proof By Minkowski's inequality for integrals we get

$$
\begin{aligned}
& P_{2 n}(M ; y)^{\frac{n-1}{n}}+P_{2 n}(N ; y)^{\frac{n-1}{n}} \\
& \quad=\left(\frac{1}{n} \int_{S^{n}}\left(\frac{1}{p_{M}(y, u)}\right)^{n} d S(u)\right)^{\frac{n-1}{n}}+\left(\frac{1}{n} \int_{S^{n}}\left(\frac{1}{p_{N}(y, u)}\right)^{n} d S(u)\right)^{\frac{n-1}{n}} \\
& \quad \geq\left(\frac{1}{n} \int_{S^{n}}\left(\frac{1}{\left.p_{M}(y, u)\right)}+\frac{1}{p_{N}(y, u)}\right)^{n} d S(u)\right)^{\frac{n-1}{n}} \\
& \quad \geq 4\left(\frac{1}{n} \int_{S^{n}}\left(\frac{1}{p_{M}(y, u)+p_{N}(y, u)}\right)^{n} d S(u)\right)^{\frac{n-1}{n}} \\
& \quad=4\left(\frac{1}{n} \int_{S^{n}}\left(\frac{1}{p_{M \tilde{+} N}(y, u)}\right)^{n} d S(u)\right)^{\frac{n-1}{n}} \\
& =4 P_{2 n}(M \tilde{+} N ; y)^{\frac{n-1}{n}}
\end{aligned}
$$

with equality if and only if $p_{M}(y, u)=p_{N}(y, u)$, that is, $M$ and $N$ are the same.

Theorem 4 Let $M_{j}(j=1, \ldots, m) \in \mathcal{S}_{o}^{n}$ and $y \in \bigcap_{j=1}^{m} M_{j}$. If $i>n$ or $n-1<i<n$, then

$$
P_{i}\left(M_{1} \tilde{+} \cdots \tilde{+} M_{n} ; y\right)^{\frac{1}{n-i}} \geq \sum_{j=1}^{m} P_{i}\left(M_{j} ; y\right)^{\frac{1}{n-i}}
$$

with equality if and only if $M_{1}, \ldots, M_{m}$ are homothetic.

If $i<n-1$, then we have

$$
P_{i}\left(M_{1} \tilde{+} \cdots \tilde{+} M_{n} ; y\right)^{\frac{1}{n-i}} \leq \sum_{j=1}^{m} P_{i}\left(M_{j} ; y\right)^{\frac{1}{n-i}}
$$

with equality if and only if $M_{1}, \ldots, M_{m}$ are homothetic. 
Proof If $i>n$ or $n-1<i<n$, then by the Minkowski linear combination and Minkowski inequality for integrals we have

$$
\begin{aligned}
P_{i}\left(M_{1} \tilde{+} \cdots \tilde{+} M_{n} ; y\right)^{\frac{1}{n-i}} & =\left(\frac{1}{n} \int_{S^{n-1}} p_{M_{1} \tilde{+} \cdots \tilde{+} M_{n}}(x, u)^{n-i} d S(u)\right)^{\frac{1}{n-i}} \\
& =\left(\frac{1}{n} \int_{S^{n-1}}\left(\sum_{j=1}^{m} p_{M_{i}}(y, u)^{n-i} d S(u)\right)\right)^{\frac{1}{n-i}} \\
& \geq \sum_{j=1}^{m}\left(\frac{1}{n} \int_{S^{n-1}} p_{M_{i}}(y, u)^{n-i} d S(u)\right)^{\frac{1}{n-i}} \\
& =\sum_{j=1}^{m} P_{i}\left(M_{j} ; y\right)^{\frac{1}{n-i}}
\end{aligned}
$$

where equality holds (by Minkowski's inequality for integrals) if $M_{1}, \ldots, M_{m}$ are homothetic.

Similarly, we can prove the case of $i<n-1$ with the reverse inequality, which follows by the Minkowski's inequality for integrals.

Particularly, if $i=2 n$, then by Theorem 4 we have the following corollary.

Corollary 4.1 Let $M_{j}(j=1, \ldots, m) \in \mathcal{S}_{o}^{n}$ and $y \in \bigcap_{j=1}^{m} M_{j}$. Then

$$
P_{2 n}\left(M_{1} \tilde{+} \cdots \tilde{+} M_{n} ; y\right)^{\frac{-1}{n}} \geq \sum_{j=1}^{m} P_{2 n}\left(M_{j} ; y\right)^{\frac{-1}{n}}
$$

with equality if and only if $M_{1}, \ldots, M_{m}$ are homothetic.

Theorem 5 Let $K_{j}(j=1, \ldots, m) \in \mathcal{K}_{o}^{n}$ and $y \in \bigcap_{j=1}^{m} K_{j}$. If $i>n$ or $1<i<n$, then

$$
P_{i}\left(K_{1} \sharp \cdots \sharp K_{n} ; y\right)^{\frac{n-1}{n-i}} \geq \sum_{j=1}^{m} P_{i}\left(K_{j} ; y\right)^{\frac{n-1}{n-i}}
$$

with equality if and only if $K_{1}, \ldots, K_{m}$ are homothetic.

If $i<1$, then we have

$$
P_{i}\left(K_{1} \sharp \cdots \sharp K_{n} ; y\right)^{\frac{n-1}{n-i}} \leq \sum_{j=1}^{m} P_{i}\left(K_{j} ; y\right)^{\frac{n-1}{n-i}}
$$

with equality if and only if $K_{1}, \ldots, K_{m}$ are homothetic.

Proof If $i>n$ or $1<i<n$, then by the definition of Blaschke linear combination and Minkowski's inequality for integrals we have

$$
\begin{aligned}
P_{i} & \left(K_{1} \sharp \cdots \sharp K_{n} ; y\right)^{\frac{n-1}{n-i}} \\
& =\left(\frac{1}{n} \int_{S^{n-1}} p_{K_{1} \sharp \cdots \sharp K_{n}}(y, u)^{n-i} d S(u)\right)^{\frac{n-1}{n-i}}
\end{aligned}
$$




$$
\begin{aligned}
& =\left(\frac{1}{n} \int_{S^{n-1}}\left(\left(p_{K_{1}}(y, u)^{n-1}+\cdots+p_{K_{1}}(y, u)^{n-i}\right)^{\frac{n-1}{n-i}} d S(u)\right)\right)^{\frac{n-1}{n-i}} \\
& \geq \sum_{j=1}^{m}\left(\frac{1}{n} \int_{S^{n-1}}\left(p_{K_{j}}(y, u)^{n-i} d S(u)\right)\right)^{\frac{n-1}{n-i}} \\
& =\sum_{j=1}^{m} P_{i}\left(K_{j} ; y\right)^{\frac{n-1}{n-i}},
\end{aligned}
$$

where equality holds (by Minkowski's inequality for integrals) if $K_{1}, \ldots, K_{m}$ are homothetic. Similarly, if $i<1$, then we can get that the reverse inequality, which follows by the Minkowski inequality for integrals.

\section{Competing interests}

The authors declare that they have no competing interests.

\section{Authors' contributions}

YY carried out inequalities of dual quermassintegrals. LZ and MZ carried out inequalities for the chord integral of a star body. All authors read and approved the final manuscript.

\section{Author details \\ 'School of Mathematics and Statistics, Southwest University, Beibei, Chongqing, 400715, P.R. China. ${ }^{2}$ College of Mathematics and Statistics, Chongqing University, Shapingba, Chongqing, 401331, P.R. China. ${ }^{3}$ Department of Financial, Southwest University, Beibei, Chongqing, 400715, P.R. China.}

\section{Acknowledgements}

Authors would like to thank two anonymous referees for many helpful comments and suggestions that directly led to the improvement of the original manuscript. Supported by Natural Science Foundation of China (Grant Nos. 11171364:

11271301; 11471266; 11426182); by 'Fundamental Research Funds for the Central Universities' (Grant Nos. XDJK2016B037; SWU115052; SWU1509190); by Natural Science Foundation Project of CQ CSTC (Grant Nos. cstc2014jcyjA00010; 2010BB9206) and by the Project-sponsored by SRF for ROCS, SEM.

\section{Received: 17 November 2015 Accepted: 9 May 2016 Published online: 01 June 2016}

\section{References}

1. Gardner, R: On the Busemann-Petty problem concerning central sections of centrally symmetric convex bodies. Bull. Am. Math. Soc. 30, 222-226 (1994)

2. Burago, YD, Zalgaller, VA: Geometric Inequalities. Springer, Berlin (1988)

3. Schneider, R: Convex Bodies: The Brunn-Minkowski Theory. Cambridge University Press, Cambridge (2014)

4. Gardner, R: Geometric Tomography, 2nd edn. Cambridge University Press, New York (2006)

5. Gardner, R: Intersection bodies and the Busemann-Petty problem. Trans. Am. Math. Soc. 342, 435-445 (1994)

6. Gardner, R: A positive answer to the Busemann-Petty problem in three dimensions. Ann. Math. 140, $435-447$ (1994)

7. Goodey, P, Weil, W: Intersection bodies and ellipsoids. Mathematika 42, 295-304 (1995)

8. Klain, D: Star Measures and Dual Mixed Volumes. Ph.D. thesis, MIT, Cambridge (1994)

9. Klain, D: Star valuations and dual mixed volumes. Adv. Math. 121, 80-101 (1996)

10. Lutwak, E: Intersection bodies and dual mixed volumes. Adv. Math. 71, 232-261 (1988)

11. Lutwak, E: Centroid bodies and dual mixed volumes. Proc. Lond. Math. Soc. 60, 365-391 (1990)

12. Zhang, G: Centered bodies and dual mixed volumes. Trans. Am. Math. Soc. 345, 777-801 (1994)

13. Hardy, GH, Littlewood, JE, Pólya, G: Inequalities. Cambridge University Press, London (1934) 